\title{
SÍNTESE E CARACTERIZAÇÃO DE NANOCOMPÓSITOS DE POLIESTIRENO/HIDROXISSAL LAMELAR
}

\author{
Samara Boaventura de Moraes, Rodrigo Botan e Liliane Maria Ferrareso Lona* \\ Departamento de Engenharia de Materiais e de Bioprocessos, Faculdade de Engenharia Química, Universidade Estadual de \\ Campinas, Av. Albert Einstein, 500, 13083-970 Campinas - SP, Brasil
}

Recebido em 14/02/2013; aceito em 21/08/2013; publicado na web em 24/09/2013

\begin{abstract}
SYNTHESIS AND CHARACTERIZATION OF POLYSTYRENE/LAYERED HYDROXIDE SALT NANOCOMPOSITES. Polystyrene/layered hydroxide salt (LHS) modified with sodium dodecyl sulfate was synthesized by in situ polymerization. The materials synthesized were characterized by gravimetry, X-ray diffraction (XRD), thermogravimetry analyses (TGA), differential scanning calorimetry (DSC) and the flammability test (FT). XRD demonstrated that synthesized nanocomposites in all compositions studied showed poor global dispersion of LHS in polystyrene. TGA showed a slight decrease in thermal stability. DSC curves showed that the glass transition temperature of polystyrene and nanocomposites were similar. The FT showed that the nanocomposite with low load of LHS exhibited good results.
\end{abstract}

Keywords: polystyrene; nanocomposites; layered hydroxide salt.

\section{INTRODUÇÃO}

Os nanocompósitos são materiais em que pelo menos um de seus componentes apresentam dimensões nanométricas. O interesse pelos nanocompósitos tem crescido acentuadamente, uma vez que estes compostos tendem a apresentar melhores propriedades quando comparados com os compósitos convencionais. A melhora nas propriedades do material ocorre devido ao fato de que as interações na interface entre matriz/reforço tendem a aumentar em escala nanométrica e isto faz com que haja um aumento nas propriedades do material. ${ }^{1-3}$

Os nanocompósitos podem ser classificados de acordo com sua matriz, sendo nanocompósitos de matriz metálica, nanocompósitos de matriz cerâmica e nanocompósitos de matriz polimérica. ${ }^{4}$

Entre os diferentes tipos de nanocompósitos poliméricos, aqueles reforçados com compostos lamelares são particularmente interessantes. Os hidroxissais lamelares (HSLs) são exemplos de compostos lamelares estudados para a síntese de nanocompósitos poliméricos.

A estrutura dos HSLs derivam da modificação da estrutura da brucita, constituída pelo hidróxido de magnésio $\left(\mathrm{Mg}(\mathrm{OH})_{2}\right)$, em que os íons $\mathrm{Mg}^{2+}$ são substituídos por íons divalentes $\left(\mathrm{Zn}^{2+}, \mathrm{Cu}^{2+}\right.$, etc.) e uma fração do grupo hidróxido é substituida por moléculas de água e ânions, como o $\mathrm{NO}_{3}{ }^{-}, \mathrm{CO}_{3}{ }^{2-}$, etc. Os HSLs apresentam a seguinte fórmula geral: $\mathrm{M}^{2+}(\mathrm{OH})_{2-\mathrm{x}}\left(\mathrm{A}^{\mathrm{m}-}\right)_{\mathrm{x} / \mathrm{m}} \cdot \mathrm{nH}_{2} \mathrm{O}$, na qual $\mathrm{M}^{2+}$ é um cátion metálico e A é um ânion (contra íon) com carga m-.$^{5}$

Diversos métodos têm sido utilizados para a síntese de nanocompósitos poliméricos, entre esses métodos destacam-se a polimerização intercalativa in situ; intercalação do polímero a partir da solução; mistura direta do polímero e das partículas e processo sol-gel., ${ }^{4,6-8}$ Dentre essas técnicas a polimerização intercalativa in situ é muito interessante, pois possibilita uma dispersão mais homogênea do reforço na matriz polimérica. ${ }^{9,10}$

Para a síntese do nanocompósito polimérico com o hidroxissal lamelar (HSL) deve ser levada em consideração a natureza hidrofílica do HSL, portanto faz-se necessária uma modificação química no HSL para torná-lo com caráter hidrofóbico e consequentemente apresentar afinidade com o polímero. A modificação ocorre por meio de reação de intercalação com ânions orgânicos. Esses ânions orgânicos fazem

\footnotetext{
*e-mail: liliane@feq.unicamp.br
}

com que se aumente o espaçamento basal, facilitando a inserção do monômero entre as lamelas do HSL. ${ }^{11-13}$

Neste trabalho foram sintetizados nanocompósitos de poliestireno e HSL, sendo esse HSL o hidroxinitrato de zinco, definido como (HNZ), este HNZ foi modificado com dodecilsulfato de sódio (HNZ/ DDS) com objetivo de melhorar sua afinidade com o estireno. Os materiais produzidos foram caracterizados por técnicas de análise gravimétrica, de difração de raios X (DRX), análise termogravimétrica (TGA), calorimetria exploratória diferencial (DSC) e teste de inflamabilidade.

\section{PARTE EXPERIMENTAL}

\section{Materiais}

Na síntese do HSL os materiais utilizados foram nitrato de zinco hexaidratado $\left(\mathrm{Zn}\left(\mathrm{NO}_{3}\right)_{2} \cdot 6 \mathrm{H}_{2} \mathrm{O}\right)$ (Synth), dodecilsulfato de sódio (DDS) $\left(\mathrm{C}_{12} \mathrm{H}_{25} \mathrm{NaO}_{4}\right)$ (Synth) e hidróxido de sódio ( $\left.\mathrm{NaOH}\right)$ (Synth).

Para a síntese dos nanocompósitos foram utilizados hidróxido de sódio $(\mathrm{NaOH})\left(\right.$ Synth), cloreto de cálcio $\left(\mathrm{CaCl}_{2}\right)$ (Ecibra), monômero (estireno 99\%) (Sigma-Aldrich) e iniciador tert-butilperóxido-2-etilhexil carbonato (TBEC 95\%) (Sigma-Aldrich).

Todos os reagentes foram utilizados em grau analítico.

\section{Síntese do hidroxissal lamelar}

O hidroxinitrato de zinco (HNZ) foi sintetizado pelo método de co-precipitação. ${ }^{5}$ Preparou-se uma solução de nitrato de zinco hexaidratado em água deionizada, a seguir o pH da solução foi ajustado por meio da adição lenta de uma solução de $\mathrm{NaOH}$, sob forte agitação e temperatura ajustada em $45^{\circ} \mathrm{C}$. O sólido formado foi lavado com água deionizada e centrifugado, em seguida foi seco em estufa a $60{ }^{\circ} \mathrm{C}$ até peso constante.

Para o HNZ/DDS, à solução de sal foi adicionada quantidade desejada do DDS, essa quantidade foi adicionada em excesso para garantir que ocorreu a troca iônica.

\section{Síntese dos nanocompósitos poliméricos}

Os nanocompósitos foram sintetizados por polimerização em 
massa via radical livre. Foram sintetizados o poliestireno puro e os nanocompósitos formados por poliestireno - HNZ/DDS (PS HNZ/ DDS) com diferentes frações mássicas (1,0; 2,0 e 3,0\%).

O primeiro passo do procedimento experimental da polimerização é a lavagem do monômero para retirar o inibidor adicionado pelo fabricante. O monômero foi lavado por uma solução de $\mathrm{NaOH} 10 \%$ e com água deionizada, em seguida foi seco com $\mathrm{CaCl}_{2}$.

Na polimerização do poliestireno puro foram utilizados monômero e iniciador, e para a polimerização dos nanocompósitos, além do monômero e iniciador, foi utilizado o HNZ/DDS. A concentração utilizada de iniciador foi de $0,0029 \mathrm{~mol} / \mathrm{L}$.

As reações de polimerização foram realizadas em ampolas. A solução contendo monômero e iniciador, ou monômero, iniciador e HNZ/DDS foi colocada em ampolas, e essas ampolas foram conectadas em uma linha de vácuo e submersas em um recipiente contendo nitrogênio líquido. Após total congelamento da solução, as válvulas foram abertas e, depois de alguns minutos, as linhas individuais de vácuo foram fechadas e uma ampola de cada vez foi retirada e lavada com etanol para descongelamento. Este procedimento se repetiu por 3 vezes. Esta etapa foi realizada com o intuito de retirar todo o oxigênio presente na amostra. Em seguida as ampolas foram seladas com auxílio de um maçarico e colocadas em um banho com circulação de fluido, a uma temperatura de $125^{\circ} \mathrm{C}$ durante 4 horas. Decorridas 4 horas de reação, as ampolas foram retiradas do banho e colocadas em um recipiente com água e gelo, para parar a reação. Em seguida as ampolas foram quebradas para retirada do material, que foi identificado e guardado para posterior caracterização.

\section{Técnicas de caracterização}

Por meio da análise gravimétrica foi obtido o perfil de conversão do estireno para o polímero puro e para os nanocompósitos, utilizando TBEC ( $0,0029 \mathrm{~mol} / \mathrm{L})$ como iniciador. A temperatura e o tempo de polimerização utilizados foram $125^{\circ} \mathrm{C}$ e 4 horas, respectivamente. Esta análise foi utilizada com o objetivo de verificar uma possível influência da presença do HSL na velocidade de polimerização.

Para as análises DRX, o equipamento utilizado foi da Shimadzu, modelo XRD 7000, e os parâmetros utilizados foram velocidade de varredura de $2^{\circ}$ a cada minuto e faixa varrida de $(2 \theta) 1,5-70^{\circ}$. O equipamento utiliza fonte com potência de $40 \mathrm{kV}$, corrente de $30 \mathrm{~mA}$ e um cátodo de cobre (1,5406 $\AA$ ).

As análises de TGA foram realizadas em um equipamento da TA Instruments, modelo TGA 2050. Os parâmetros utilizados foram velocidade de aquecimento de $20^{\circ} \mathrm{C} / \mathrm{min}$ em uma faixa de temperatura de $25-700{ }^{\circ} \mathrm{C}$. As análises foram realizadas em atmosfera inerte (fluxo de gás argônio $100 \mathrm{ml} / \mathrm{min}$ ).

Para as análises de DSC, foi utilizado um equipamento da Mettler-Toledo, modelo DSC 823e. Os parâmetros utilizados foram velocidade de aquecimento de $20^{\circ} \mathrm{C} / \mathrm{min}$ em uma faixa de temperatura de $25-300{ }^{\circ} \mathrm{C}$. As análises foram realizadas em atmosfera inerte (fluxo de nitrogênio líquido $50 \mathrm{ml} / \mathrm{min}$ ). Foi utilizada análise dinâmica, com aquecimento de 25 a $300{ }^{\circ} \mathrm{C}$, resfriamento de 300 a $25{ }^{\circ} \mathrm{C}$ e novamente aquecimento de 25 a $300{ }^{\circ} \mathrm{C}$.

Para o teste de inflamabilidade, foi utilizado o teste da UL 94 $\mathrm{HB}$, com modificação no comprimento do corpo de prova de 125 para $64 \mathrm{~mm}$. O teste foi realizado com corpos de prova na horizontal, que foram preparados por injeção em uma mini injetora da marca ThermoScientific, modelo Haake Mini Jet II.

\section{RESULTADOS E DISCUSSÃO}

A Figura 1 mostra os resultados obtidos na conversão do estireno para o polímero puro e para os nanocompósitos (PS HNZ/DDS) por meio da análise gravimétrica. Como pode ser observado, há pequena variação na conversão dos nanocompósitos quando comparados com o poliestireno puro. Ao fim da reação $(4 \mathrm{~h})$ o poliestireno puro apresenta conversão de $98 \%$, enquanto que os nanocompósitos apresentam conversão média de $95 \%$, ou seja, praticamente não há diferença na conversão.

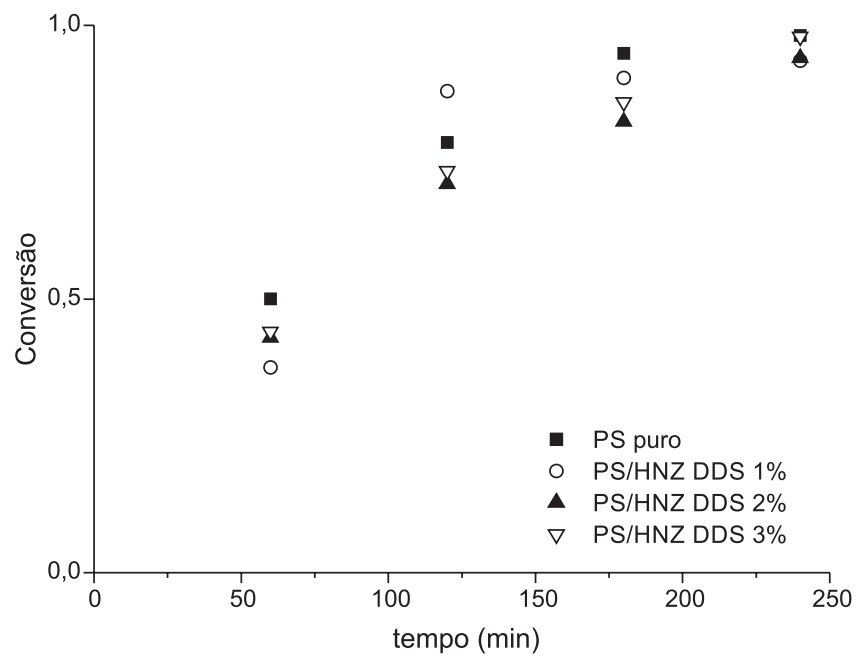

Figura 1. Comparação entre os resultados obtidos na conversão do polímero puro e dos nanocompósitos PS HNZ/DDS

Em algumas reações de polimerizações, a presença de reforços no sistema reacional, como argilas ou hidróxidos duplos lamelares, pode acelerar ou retardar a velocidade de reação. Pela Figura 1, observa-se que a presença do HSL proposto neste trabalho não afeta a velocidade da reação, sendo as variações observadas da mesma ordem de magnitude que os erros experimentais característicos da análise gravimétrica.

As análises de DRX (Figura 2) permitiram calcular o espaçamento basal e o espaçamento interlamelar do HNZ/DDS sintetizado, e comparar o difratograma do poliestireno puro com os difratogramas dos nanocompósitos.

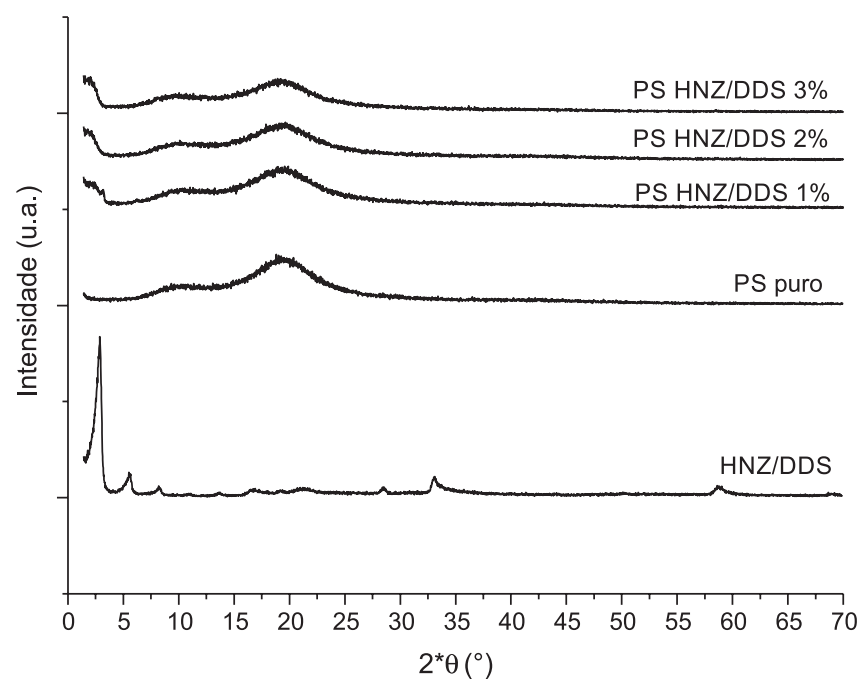

Figura 2. Difratogramas do HNZ/DDS, PS puro e dos nanocompósitos PS $H N Z / D D S$

O espaçamento basal do HNZ/DDS foi calculado através da Equação de Bragg ( $n=2 d \operatorname{sen} \theta)$. Para o cálculo utilizou-se o pico de maior intensidade do difratograma do HNZ/DDS. O valor do 
espaçamento basal para o HNZ/DDS sintetizado é 29,62 Å. A partir do espaçamento basal, é possível obter o valor do espaçamento interlamelar retirando o valor da lamela da brucita, que é $4,8 \AA$, sendo o valor do espaçamento interlamelar igual a $24,82 \AA$. Sabe-se da literatura que o valor do espaçamento basal do HNZ é de aproximadamente $9,78 \AA{ }^{14}$ portanto pode-se afirmar que houve a modificação no HNZ/ DDS sintetizado, uma vez que este apresentou espaçamento basal superior ao HNZ. A diferença no espaçamento basal obtida entre o HNZ e o HNZ/DDS foi de 19,84 A. Este resultado apresenta uma boa consistência, uma vez que estudos indicam que o tamanho da cadeia individual do DDS é 20,7 , valor muito próximo ao obtido neste trabalho. Esta pequena diferença existente entre os valores pode ser atribuída à interação dos ânions DDS com as lamelas do HSL. ${ }^{15,16}$

$\mathrm{O}$ aumento no espaço interlamelar diminui as interações entre as lamelas, facilitando a inserção do monômero entre elas, o que possibilita a formação do nanocompósito. ${ }^{17}$

O poliestireno, sendo um polímero amorfo, não apresenta nenhum pico significativo na DRX, apenas pequenos desvios da linearidade (halos) que representam uma pequena porção de orientação do material. Como esperado, o poliestireno não apresenta ordem de longo alcance. ${ }^{15,18}$

Como pode ser observado, os nanocompósitos apresentam difratogramas semelhantes ao do poliestireno puro, com desvios da linearidade na faixa de $10^{\circ}$ e $20^{\circ}(2 \theta)$, assim como observado para o poliestireno puro. Estes nanocompósitos também possuem pequenos picos na região de $2,5-3,0^{\circ}(2 \theta)$, que são referentes ao pico de maior intensidade do HNZ/DDS $\left(2,98^{\circ}\right)(2 \theta)$. Este pico na região de $2,5-3,0^{\circ}$ (20) corresponde a um espaçamento basal de $35 \AA$, ou seja, maior que o espaçamento basal do HNZ/DDS $(29,62 \AA)$, sugerindo que as cadeias poliméricas estejam intercaladas entre as lamelas do HSL.

Para verificar o perfil térmico do poliestireno puro e dos nanocompósitos produzidos foram realizadas TGA. As curvas da TGA são apresentadas na Figura 3.

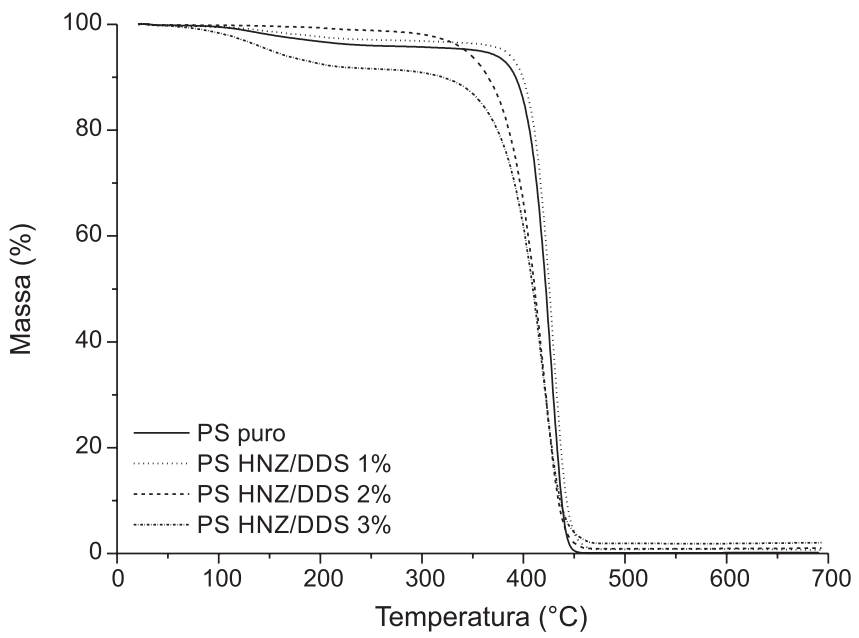

Figura 3. Termogramas do PS puro e dos nanocompósitos PS HNZ/DDS

O poliestireno puro tem uma leve perda de massa por volta de $115^{\circ} \mathrm{C}$. Esta perda provavelmente está relacionada à perda de água presente na estrutura do poliestireno. Entre $120-360{ }^{\circ} \mathrm{C}$ a massa se mantém constante e, a partir deste ponto, ocorre a degradação das cadeias poliméricas até aproximadamente $443{ }^{\circ} \mathrm{C}$, temperatura na qual praticamente todo o material já foi consumido.

Os nanocompósitos com 1,0 e 3,0\% de agente de reforço apresentam uma perda de massa na faixa de $96-112^{\circ} \mathrm{C}$, relacionada à perda de água presente na estrutura do HSL. Já o nanocompósito com $2,0 \%$ de agente de reforço manteve-se constante até aproximadamente $300{ }^{\circ} \mathrm{C}$.
Para todos os nanocompósitos a degradação das cadeias poliméricas ocorre na faixa de $300-445^{\circ} \mathrm{C}$, sendo que em $445^{\circ} \mathrm{C}$ basicamente todo o material já havia sido degradado.

Comparando os resultados obtidos para o poliestireno puro e os nanocompósitos, verifica-se que há uma leve queda na estabilidade térmica dos nanocompósitos em relação ao poliestireno puro. Apenas o nanocompósito com $1,0 \%$ de agente de reforço apresentou uma pequena melhora na estabilidade térmica. Quando comparados à temperatura de $10 \%$ de perda de massa, verifica-se que houve um acréscimo de $5{ }^{\circ} \mathrm{C}$ na temperatura em relação ao poliestireno puro. Com $50 \%$ de perda de massa, há um aumento de $2{ }^{\circ} \mathrm{C}$ na temperatura e, finalmente, na temperatura final de decomposição, observa-se um aumento de $5{ }^{\circ} \mathrm{C}$ na temperatura final de decomposição do nanocompósito em relação ao poliestireno puro. Esta leve melhora na estabilidade térmica do PS HNZ/DDS 1,0\% pode estar relacionada a uma melhor dispersão do HNZ/DDS na matriz polimérica deste nanocompósito, em comparação aos demais nanocompósitos.

A temperatura de transição vítrea do poliestireno puro e dos nanocompósitos foram determinadas por calorimetria exploratória diferencial (DSC). Na Figura 4 estão representadas as curvas de DSC obtidas.

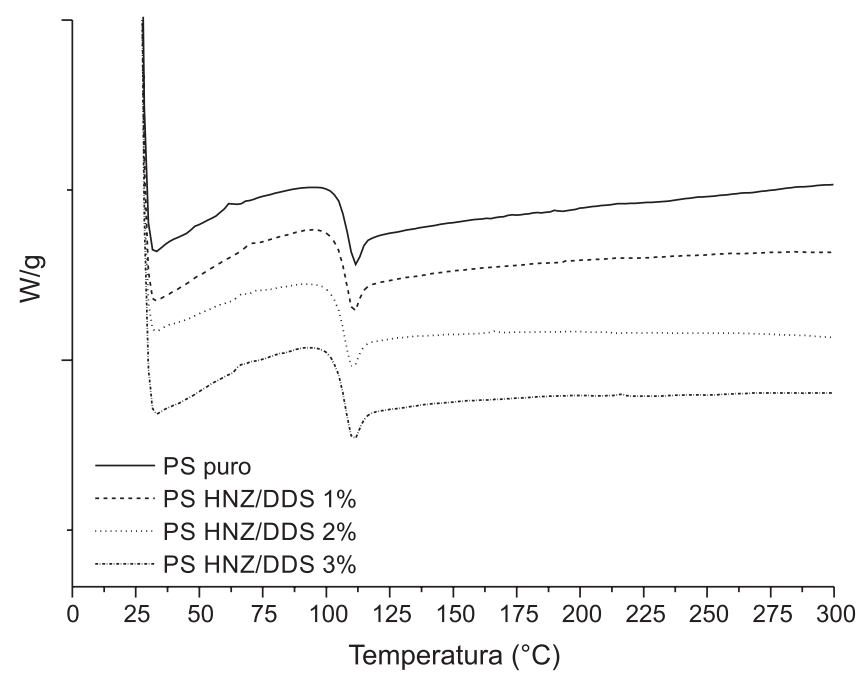

Figura 4. Curvas de DSC do PS puro e dos nanocompósitos PS HNZ/DDS

A temperatura de transição vítrea obtida para o poliestireno puro foi de $107,04{ }^{\circ} \mathrm{C}$, enquanto que para os nanocompósitos com 1,0 , 2,0 e $3,0 \%$ de agente de reforço foram $105,02^{\circ} \mathrm{C}, 106,50{ }^{\circ} \mathrm{C}, 106,02$ ${ }^{\circ} \mathrm{C}$ e $106,02^{\circ} \mathrm{C}$, respectivamente. Os nanocompósitos apresentaram temperatura de transição vítrea semelhante à do poliestireno puro, indicando que o HSL pouco influencia no processamento dos nanocompósitos.

Com o objetivo de avaliar o grau de queima dos nanocompósitos em relação ao poliestireno puro, foi realizado o teste de inflamabilidade baseado no teste da UL 94 HB. ${ }^{19}$ Os resultados obtidos estão representados na Figura 5.

Os resultados obtidos com o teste de inflamabilidade mostram que os nanocompósitos com 1,0\% de agente de reforço queimam menos que o poliestireno puro, enquanto que os nanocompósitos com 2,0 e 3,0\% de agente de reforço queimam mais que o poliestireno puro. Observa-se na Figura 5 que o poliestireno puro apresenta grau de queima de aproximadamente $0,8 \mathrm{~mm} / \mathrm{s}$, enquanto que os nanocompósitos PS HNZ/DDS 1,0\% apresentaram grau de queima de aproximadamente $0,5 \mathrm{~mm} / \mathrm{s}$, apresentando efeito de barreira. Já os nanocompósitos PS HNZ/DDS 2,0 e 3,0\% apresentaram grau de queima superior ao do poliestireno (na faixa de $0,95 \mathrm{~mm} / \mathrm{s}$ ), indicando 


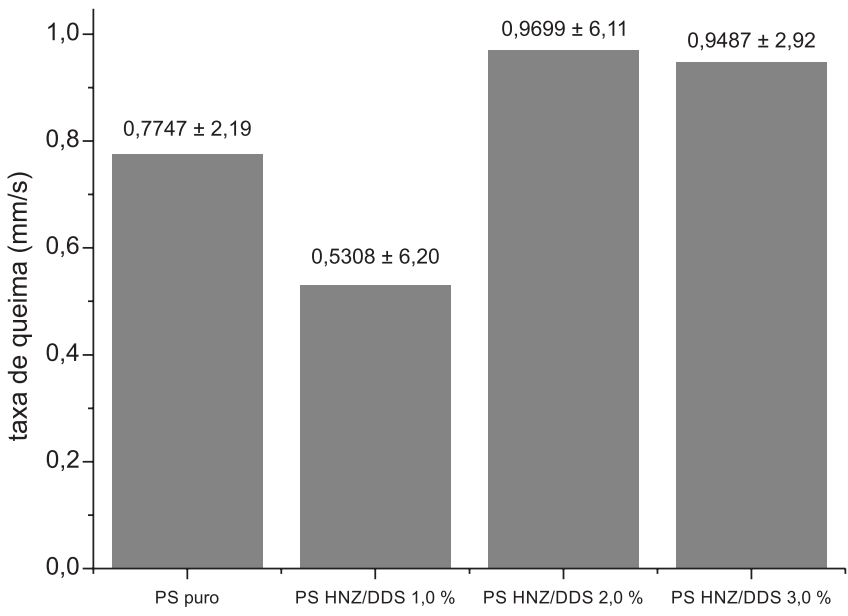

Figura 5. Teste de inflamabilidade do PS puro e dos nanocompósitos PS HNZ/DDS

um efeito catalisador na queima, ou seja, acelera o processo de queima do material..$^{20}$

\section{CONCLUSÕES}

Foram sintetizados nanocompósitos poliméricos de poliestireno/ hidroxinitrato de zinco modificado com dodecilsulfato de sódio. As análises de DRX mostraram que o HSL sintetizado realmente foi modificado com o DDS, apresentando espaçamento basal maior que o do hidroxinitrato de zinco sem modificação.

A análise gravimétrica mostrou que a curva de conversão do poliestireno puro e dos nanocompósitos PS HNZ/DDS são muito semelhantes.

Os nanocompósitos apresentaram estabilidade térmica inferior ao do poliestireno puro, exceto para o nanocompósito com $1,0 \%$ de agente de reforço, que apresentou uma pequena melhora em sua estabilidade térmica quando comparada ao poliestireno puro.

As análises de DSC permitiram avaliar a temperatura de transição vítrea do poliestireno e dos nanocompósitos. Os nanocompósitos apresentaram temperatura de transição vítrea próxima ao do poliestireno, indicando que a presença do HSL pouco influencia no processamento do polímero.

E por fim, o teste de inflamabilidade realizado mostrou que os nanocompósitos com menor carga de HSL (1,0\%) tendem a apresentar efeito de barreira nos nanocompósitos produzidos, retardando a queima do material quando comparados com o poliestireno puro.

\section{AGRADECIMENTOS}

À FAPESP (2011/05452-1) pela bolsa de mestrado concedida e a Faculdade de Engenharia Química - UNICAMP pela infraestrutura disponibilizada.

\section{REFERÊNCIAS}

1. Botan, R.; Nogueira, T. R.; Lona, L. M. F.; Wypych, F.; Polímeros 2011 , 21,34 .

2. Souza, M. A.; Pessan, L. A.; Rodolfo Junior, A.; Polímeros 2006, 16, 257.

3. Nogueira, T.; Botan, R.; Wypych, F.; Lona, L.; Composites Part A 2011, 42, 1025.

4. Camargo, P. H. C.; Satyanarayana, K. G.; Wypych, F.; Mater. Res. 2009, 12,1 .

5. Arizaga, G. G. C.; Satyanarayana, K. G.; Wypych, F.; Solid State Ionics 2007, 178, 1143.

6. Alexandre, M.; Dubois, P.; Mater. Sci. Eng. 2000, $28,1$.

7. Pavlidou, S.; Papaspyrides, C. D.; Prog. Polym. Sci. 2008, 33, 1119.

8. Yano, K.; Usuki, A.; Okada, A.; Kurauchi, T.; Kamigaito, O.; J. Polym. Sci., Part A: Polym. Chem. 1993, 31, 2493.

9. Esteves, A. C. C.; Barros-Timmons, A.; Trindade, T.; Quim. Nova 2004, 27, 798.

10. Nogueira, T.; Botan, R.; Wypych, F.; Lona, L.; J. Appl. Polym. Sci. 2012, 124, 1764.

11. Vaia, R. A.; Teukolsky, R. K.; Giannelis, E. P.; Chem. Mater. 1994, 6, 1017.

12. Avalos, F.; Ortiz, J. C.; Zitzumbo, R.; López-Manchado, M. A.; Verdejo, R.; Arroyo, M.; Eur. Polym. J. 2008, 44, 3108.

13. Leite, I. F.; Malta, O. L.; Raposo, C. M. O.; Canedo, L. E.; Carvalho, L. H.; Silva, S. M. L.; Polímeros 2011, 21, 195.

14. Marangoni, R.; Ramos, L. P.; Wypych, F.; J. Colloid Interface Sci. 2009, 330, 303.

15. Botan, R.; Nogueira, T. R.; Wypych, F.; Lona, L. M. F.; Polym. Eng. Sci. 2012, 52, 1754.

16. Kotal, M.; Kuila, T., Srivastava, S. K.; Bhowmick, A. K.; J. Appl. Polym. Sci. 2009, 114, 2691.

17. Qiu, L.; Chen, W.; Qu, B.; Colloid Polym. Sci. 2005, 283, 1241.

18. Qiu, L.; Chen, W.; Qu, B.; Polym. Degrad. Stab. 2005, 87, 433.

19. http://www.boedeker.com/bpi-ul94.htm, acessada em Setembro 2013.

20. Nogueira, T.; Botan, R.; Neto, J. C. M.; Wypych, F.; Lona, L.; Adv. Polym. Tech. 2013, 32, E660. 